\title{
QUEEN'S
UNIVERSITY
BELFAST
}

\section{Evaluation of Direct from Sample Metabolomics of Human Faeces using Rapid Evaporative lonisation Mass Spectrometry (REIMS)}

Cameron, S. J. S., Alexanders, J. L., Bolt, F., Burke, A., Ashrafian, H., Teare, J. P., Marchesi, J. R., Kinross, J. M., Li, J. V., \& Takats, Z. (2019). Evaluation of Direct from Sample Metabolomics of Human Faeces using Rapid Evaporative Ionisation Mass Spectrometry (REIMS). Analytical Chemistry, 91(21), 13448.

https://doi.org/10.1021/acs.analchem.9b02358

Published in:

Analytical Chemistry

Document Version:

Peer reviewed version

Queen's University Belfast - Research Portal:

Link to publication record in Queen's University Belfast Research Portal

Publisher rights

(c) 2019 American Chemical Society. This work is made available online in accordance with the publisher's policies. Please refer to any applicable terms of use of the publisher.

\section{General rights}

Copyright for the publications made accessible via the Queen's University Belfast Research Portal is retained by the author(s) and / or other copyright owners and it is a condition of accessing these publications that users recognise and abide by the legal requirements associated with these rights.

Take down policy

The Research Portal is Queen's institutional repository that provides access to Queen's research output. Every effort has been made to ensure that content in the Research Portal does not infringe any person's rights, or applicable UK laws. If you discover content in the Research Portal that you believe breaches copyright or violates any law, please contact openaccess@qub.ac.uk. 
1 Evaluation of Direct from Sample Metabolomics of Human Faeces using Rapid Evaporative

2 Ionisation Mass Spectrometry (REIMS)

3

4 Simon J.S. Cameron (1)†, James L. Alexander (1)†, Frances Bolt (1), Adam Burke (1), Hutan Ashrafian

5 (1), Julian Teare (1), Julian R. Marchesi (1,2), James Kinross (1), Jia V. Li (1), Zoltán Takáts (1)*

6

7 (1) Department of Surgery and Cancer, Imperial College London, London, SW7 2AZ, UK.

8 (2) School of Biosciences, Cardiff University, Cardiff, CF10 3AX, UK.

9

10 + Contributed equally.

11

$12 *$ Corresponding Author: Professor Zoltan Takáts, Division of Computational and Systems Medicine,

Department of Surgery and Cancer, Imperial College London, South Kensington Campus, Sir Alexander

Fleming Building, London, SW7 2AZ. Telephone: 0207594 2760. Email: z.takats@imperial.ac.uk 
Abstract

Mass spectrometry is a powerful tool in the investigation of the human faecal metabolome. However, current approaches require time-consuming sample preparation, chromatographic separations, and consequently long analytical run times. Rapid evaporative ionisation mass spectrometry (REIMS) is a method of ambient ionisation mass spectrometry and has been utilised in the metabolic profiling of a diverse range of biological materials, including human tissue, cell culture lines, and microorganisms. Here, we describe the use of an automated, high-throughput REIMS robotic platform for direct analysis of human faeces. Through the analysis of faecal samples from five healthy male participants, REIMS analytical parameters were optimised and used to assess the chemical information obtainable using REIMS. Within the faecal samples analysed, bile acids, including primary, secondary, and conjugate species were identified, and phospholipids of possible bacterial origin were detected. In addition, the effect of storage conditions and consecutive freeze/thaw cycles was determined. Within the REIMS mass spectra, the lower molecular weight metabolites, such as fatty acids, were shown to be significantly affected by storage conditions for prolonged periods at temperatures above $-80^{\circ} \mathrm{C}$, and consecutive freeze/thaw cycles. However, the complex lipid region was shown to be unaffected by these conditions. A further cohort of 50 faecal samples, collected from patients undergoing bariatric surgery, were analysed using the optimised REIMS parameters, and the complex lipid region mass spectra used for multivariate modelling. This analysis showed a predicted separation between pre- and post-surgery specimens, suggesting that REIMS analysis can detect biological differences, such as microbiome-level differences, which have traditionally been reliant upon methods utilising extensive sample preparations and chromatographic separations and/or DNA sequencing. 
Mass spectrometry is a powerful and widely used tool for the metabolic profiling of biological fluids and tissues. It is capable of generating extensive information on the metabolic composition of a sample, which allows for interrogation of metabolic function, host-microbiome dysbiosis, response to external stimuli and stress, and biomarker discovery for disease ${ }^{1}$. As a result of its ease of sampling, the analysis of human faeces is commonplace for the study of the gut microbiome. It is also at the interface of the cross-talk between the gut microbiome and the host, which is involved in the regulation of a number of host metabolic pathways ${ }^{2}$. However, many studies look solely at the taxonomic composition of the microbes in a gut microbiome, meaning insightful metabolomic data is overlooked ${ }^{3,4}$. In the study of the human metabolome, samples such as urine and blood are frequently used; primarily as a result of their ease of collection and rich content of metabolites ${ }^{5-7}$. However, alternative sample types show promise as a source of disease biomarkers, particularly those which are at the interface of disease onset, such as sputum or exhaled breath for lung cancer ${ }^{8}$, and saliva for oral cancers ${ }^{9}$. Human faeces has received particular attention in recent years as a source of disease biomarkers, primarily due to its ease and non-invasive method of collection, and localisation to affected gastrointestinal diseases sites, such as colorectal cancer ${ }^{10}$ and inflammatory bowel disease ${ }^{11}$.

Mass spectrometric techniques and platforms which are commonly used in the metabolic profiling of human faeces include liquid chromatography-mass spectrometry (LC-MS) ${ }^{12}$ and gas chromatographymass spectrometry (GC-MS) ${ }^{13}$. Both of these techniques require extensive sample preparation, such as the creation of faecal water samples, chemical derivatisation, or targeted metabolite extraction and/or removal ${ }^{14}$. The requirement for sample preparation reduces analytical throughput, is typically user intensive and unpleasant, and can result in significant chemical changes ${ }^{15}$, which may impair the ability to identify, for example, disease biomarkers. In addition, both methods are reliant on chromatographic separation before mass spectrometry analysis; which carries the benefit of allowing 
high levels of analytical resolution, but extends sample running time within the range of approximately ten to 20 minutes.

In recent years, the field of ambient ionisation mass spectrometry has expanded rapidly, allowing mass spectrometric analysis of a biological sample with no preparative steps and under normal atmospheric conditions ${ }^{16}$. One such method is rapid evaporative ionisation mass spectrometry (REIMS); which has seen successful applications including the in-situ, real-time detection of intra-operative tumour margins ${ }^{17}$ and as a platform for the species-level classification of clinically important microorganisms directly from a culture plate ${ }^{18,19}$. REIMS works by applying a radiofrequency electrical current directly to a sample. The sample, as a result of its non-zero impedance, rapidly heats and gas phase ions of metabolites and structural lipids are released into an analyte-containing vapour, which is subsequently aspirated into a mass spectrometer, using the instrument's operational vacuum, for mass spectrometric analysis. Initial applications of REIMS utilised electrosurgical hand-pieces, such as electrical diathermy surgical devices and bipolar surgical forceps, to direct the electrical current and aspirate the analyte-containing vapour to a mass spectrometer ${ }^{20}$. Recent advances have adapted REIMS to a modified liquid handling platform, which allows for the high-throughput (approximately 34,000 samples per 24 hour period) semi-automated analysis of samples with minimal user input ${ }^{21,22}$. Although initially developed to be suitable for implementation in a high-throughput clinical microbiology diagnostic laboratory, the platform is highly versatile and capable of analysing a wide range of biological samples using a universal set-up.

In this study, we report on the first application of REIMS to the metabolic profiling of human faecal samples; showing its ability to detect bile acids and phospholipid markers of microbiome constituents. Further, we utilise the optimised REIMS parameters identified to the analysis of faecal samples from patients undergoing bariatric surgery, showing that REIMS possesses the analytical sensitivity to detect biological changes resulting from a clinical intervention. 


\section{Collection of Faecal Samples}

For REIMS analysis of human faeces, a total of five healthy male participants, with no history of gastrointestinal disease, donated a stool sample in the morning using a FECOTAINER collection device. Full local ethical approval was obtained (Research Ethics Committee reference 14/EE/0024). Participant information is given in Table S1. Of the five stool samples, two were graded as Type 3, two as Type 5, and one Type 2; according to the Bristol Stool Scale ${ }^{23}$. Within two hours of sample donation, faecal samples were separated into eleven equal aliquots and stored according to the study outline shown in Figure S1. In addition, 50 faecal samples were collected from patients undergoing bariatric surgery. Of these, 15 were collected from patients prior to undergoing surgery, 11 were collected 2-6 months post-surgery, and 24 were collected 1-2 years post-surgery. Patients were recruited from Charing Cross Hospital and Hammersmith Hospital (London, UK) under an ethics permission (Metabolic Phenotype and Modulation in Obese and Bariatric Surgical Patients 08/H0711/123).

102

REIMS Analysis of Faecal Samples

REIMS analysis was completed on all samples as previously described for the high-throughput, semiautomated analysis of microbial cultures on solid growth media ${ }^{21}$. In brief, faecal samples were placed onto the base of a sterile petri dish on a Pickolo visualisation platform, which also acted as the return electrode for REIMS monopolar analysis, connected to a modified TECAN Freedom EVO One liquid handling platform ${ }^{21,22}$. For each analysis, an area of approximately $1 \mathrm{~mm}^{2}$ is analysed with the monopolar probe penetrating approximately $0.2 \mathrm{~mm}$ into the sample. The glass surface of the visualisation platform was coated with a conductive material that allowed the transfer of the electrical current through capacitive coupling. A radiofrequency electrical current was passed through a stainless steel monopolar probe with a sharp tip and the analyte vapour channelled to a Xevo G2-XS QToF mass spectrometer via PTFE tubing. Prior to entry into the mass spectrometer, the analyte 
vapour was mixed with 2-propanol containing leucine encephalin at a concentration of $10 \mathrm{ng} / \mathrm{mL}$, at a flow rate of $0.2 \mathrm{~mL}$ per minute, in a T-piece unit. The resulting mixture passed through the REIMS atmospheric interface chamber, as previously detailed, where solvent-ion clusters collide with a heated $\left(700-800^{\circ} \mathrm{C}\right)$ coil before entry into the Stepwave ${ }^{\circledR}$ of the MS instrument. For each sample and/or analysis parameter, a total of five analytical repeats were acquired. For each analytical repeat, a single stainless steel monopolar probe was used. Probes were re-used after removal of any particulate matter and vortex cleaning in 2-propoanol. Mass spectra were collected in negative ion and positive ion detection modes using instrument parameters in Table S2 and over the $\mathrm{m} / \mathrm{z}$ range of 50 to 2500 . For each day of operation, the mass spectrometer was calibrated using sodium formate according to the manufacturer's instructions. For instrument cleaning, the disassembled T-piece unit was cleaned at the end of each running day through sonication for 10 minutes in 2-propanol. The standard laboratory routine for cleaning of the instrument was followed whereby the Stepwave ${ }^{\circledast}$ is removed after venting and sonicate cleaned in $50 \%$ methanol $(\mathrm{v} / \mathrm{v})$ made up using water for ten minutes and then in $100 \%$ methanol for a further ten minutes.

\section{Analysis of Mass Spectrometry Data and Statistical Analysis}

To correct for mass drift and remove background mass spectral signals, all .RAW data files were processed using MassLynx software (V4.1, Waters) through the Accurate Mass Measure tool employing the Automatic Peak Detection option, background subtraction, and with lock mass correction against leucine enkephalin ( 554.2615 for negative ion mode data and 556.2712 for positive ion mode data) with a mass window of $+/-0.500$, and averaged over a total of ten scans. Resulting processed centroid mass spectra were used in subsequent intensity analysis calculations, identification of most intense spectral peaks, and $\mathrm{m} / \mathrm{z}$ values for accurate mass identification of metabolites. For intensity analysis, mean and standard deviations were calculated from individual intensity values taken for five identified analytical repeats identified through total ion count chromatogram peaks. 
141 Additionally, raw mass spectral data files were processed using the Offline Model Building (OMB) software (Version 1.1.29.0, Waters) in order to perform background subtraction, mass drift correction against leucine encephalin lock mass compound (negative mode $\mathrm{m} / \mathrm{z}=554.3$ ), and mass binning to 0.1 Da within chosen restricted mass ranges. For statistical analysis, including principal component analysis (PCA), hierarchical cluster analysis (HCA), and univariate statistical analysis, the MetaboAnalyst $3.0^{24}$ platform was used. After processing of mass spectra, a data matrix was exported from the $\mathrm{OMB}$ software and uploaded to the MetaboAnalyst 3.0 online analysis pipeline where it underwent additional processing for log transformation and Pareto scaling (mean-centred and divided by the square root of standard deviation of each variable). For univariate statistical analysis, the nonparametric Kruskal-Wallis test was used with a significance threshold, after false discovery rate correction, of less than 0.05 used to identify significantly different mass bins. For PCA analysis, the first and second principal components were used in two dimensional plots, with $95 \%$ confidence regions displayed using colour shading. For HCA analysis, dendograms with heat maps were constructed using the top 25 mass bins based on univariate statistical analysis and constructed using Euclidean distance measure, and Ward clustering algorithm.

\section{Tentative Identification of Highest Intensity Phospholipids and Bile Acids}

Metabolite identification of mass spectral peaks was accomplished through two approaches. For the highest intensity phospholipids within the 600 to $1000 \mathrm{~m} / \mathrm{z}$ range, collision-induced dissociation tandem mass spectrometry was conducted using the Xevo G2-XS QToF instrument. The peaks were chosen from across the five initial samples used for heating power optimisation, using spectra obtained using the optimised heating power of $35 \mathrm{~W}$. The five participants' sample aliquot stored as shown in Figure S1 were used in tandem mass spectrometry. Before fragmentation of the target $\mathrm{m} / \mathrm{z}$ peak, the resolution of the quadrupole was optimised using the constant signal of the leucine enkephalin lock mass compound infused with 2-propanol. After optimisation to ensure that only one 
mass spectral peak was visible after the quadrupole instrument was set to target 554.26 (in negative ion detection mode), each of the targeted phospholipid $\mathrm{m} / \mathrm{z}$ values were in turn fragmented. The collision energy of the Xevo G2-XS QToF instrument was optimised for each targeted $\mathrm{m} / \mathrm{z}$ peak until the parent ion had a relative intensity value of between one third and one half of the product ion with the highest relative intensity. The resulting fragmentation patterns were used to assign identifications according to head group and acyl chain combinations.

172

For tentative identification of metabolites of interest, such as bile acids (primary, secondary, and conjugates), fatty acids, low molecular weight metabolites, and phospholipids, accurate mass measures were used. A number of negative ionisation $\mathrm{m} / \mathrm{z}$ values were identified in the acquired REIMS mass spectra. Accurate masses of candidate $\mathrm{m} / \mathrm{z}$ peaks, to two decimal places after mass drift correction, were used to interrogate the Human Metabolome Database ${ }^{25}$ and the LIPID MAPS database ${ }^{26}$, with the highest ranked match, based on Delta value $(<0.01)$, after removal of specific drug- and diet-related metabolites, taken as the tentative identification shown in Tables S3 and S4 and Supplementary Data Matrix 1.

\section{Safety Considerations}

In this work, all samples were treated as potential Hazard Group 2 material and were therefore manipulated within containment level 2 facilities. All REIMS analysis was handled within a class 2 biological safety cabinet. All solvents, such as 2-propanol and methanol, were handled according to the material safety data sheet provided by their respective manufacturer. 
Faecal samples were successfully collected from five male participants and analysed using an automated REIMS monopolar electrode platform, Figure 1. The automated set-up allowed sample analysis without pre-treatment nor extraction in approximately ten seconds. As five replicate sampling points were taken, this allowed sample analysis to be completed in approximately one minute. Initial inspection of the resulting mass spectra shows a dominance of fatty acids including saturated fatty acids, monounsaturated fatty acids, and polyunsaturated fatty acids above 13 carbons in length. In the higher mass range, between 600 and $1200 \mathrm{~m} / \mathrm{z}$, there is a lower intensity of complex lipids, typically detected using REIMS, including glycerophospholipids and diglycerides.

\section{Optimisation of Heating Power and Analytical Set-Up}

Signal intensities for each heating power within the fatty acid (50 to $500 \mathrm{~m} / \mathrm{z}$ ), and complex lipid (600 to $1000 \mathrm{~m} / \mathrm{z}$ ) regions typically seen in REIMS mass spectra, alongside a measure of instrument noise ( 50 to $51 \mathrm{~m} / \mathrm{z}$ ), are given in Figure 2a. Unsupervised principal component analysis (PCA) was completed using sample classes according to heating power, Figure $2 b$, and participant, Figure $2 c$. Within both PCA plots, high variability is seen between mass spectra acquired using a heating power of $25 \mathrm{~W}$ or lower and 40W or higher. However, such a trend is not visible with spectra acquired using a heating power of either $30 \mathrm{~W}$ or $35 \mathrm{~W}$. A similar trend is evident within signal intensities as was observed with PCA modelling. Mean signal intensities for heating powers below 30W show a trend towards decreased fatty acid and complex lipid region intensities, and 40W and above a trend towards increased fatty acid, but decreased complex lipid region signals, with an overall positive correlation between spectral noise and heating power observed. As a result of these evident trends, an optimum heating power of $35 \mathrm{~W}$ was chosen as a universal parameter suitable for the analysis of all stool types. Following heating power optimisation, a total of 169 features were given tentative identifications, across the entire mass spectral range analysed for all five participants. This figure however, represents 
only a fraction of the total features detected in the REIMS mass spectra. Representative mass spectra for analysis of all five participant samples, after background subtraction and mass drift correction, are given in Supplementary Figures S2 and S3. To determine the requirement for analytical replicates of a sample, the heterogeneity present within samples was explored for REIMS analysis. As traditional mass spectrometry approaches require sample preparation and extraction, homogenisation of the sample is typical. With REIMS analysis however, individual analytical sampling points across the sample are used. Supplementary Figure S4 shows PCA modelling of the five analytical points for the optimised 35W heating power. There appears to be minimal heterogeneity across the five analytical repeats, particularly within the 600 to $1000 \mathrm{~m} / \mathrm{z}$ range, although the analytical repeats from one regions of each sample were used for subsequent REIMS analysis.

Initial optimisation of faecal analysis using REIMS was completed in negative ion detection mode as previous studies have shown this mode provides the richest mass spectra with regards to both signal intensity and diversity of detectable ion species ${ }^{19}, 27$. Positive ion detection mode REIMS data was also collected on the five samples used in heating power optimisation using $35 \mathrm{~W}$. A representative mass spectrum is shown in Figure 1c alongside mass spectra for all five participants in Supplementary Figure S3. Principal component analysis of positive ion detection mode, Supplementary Figure S5, shows that participant differences are not observable in either the 50 to $500 \mathrm{~m} / \mathrm{z}$ nor 600 to $1000 \mathrm{~m} / \mathrm{z}$ mass ion spectra contain likely complex lipid features across a wider mass range of 600 to $1000 \mathrm{~m} / \mathrm{z}$. At the 
acid ions which are not easily distinguishable in positive ion spectra due to the higher level of analytical noise. Analysis of mass spectra in both ion detection modes indicates higher levels of analytical noise are present within the positive mode spectra which may explain the inability to separate participants in PCA. Thus, for further analysis of faecal samples, negative ion detection mass spectra were used.

\section{Effect of Sample Storage Conditions on REIMS Mass Spectra}

Following optimisation of the sample heating power, the effect of storage duration and condition on the mass spectra generated by REIMS was determined. REIMS analysis was completed on samples after normalisation to room temperature for one hour using the previously identified optimum universal heating power of $35 \mathrm{~W}$. Unsupervised PCA analysis was completed on the eight-different storage length and condition combinations and compared to mass spectra obtained from fresh faecal samples. Figure 3a shows that overall, no significant differences are evident as a result of any storage condition and time duration combination. Indeed, hierarchical cluster analysis based on the top 25 mass bins, ranked by the $P$ value of analysis of variance tests between participants, shows that participant differences are clear across all sample storage condition and duration combinations, Figure 3b. The contributing mass bins to this clear separation are primarily within the REIMS complex lipid region (600 to $1000 \mathrm{~m} / \mathrm{z}$ ). Analysis of signal intensities between storage conditions as compared to fresh faecal samples, Supplementary Figure S6, show that for all conditions, fatty acid and low molecular weight metabolites regions are significantly higher than that of the typically complex lipid region. In regard to the effect of storage conditions on signal intensities, it is clear that the complex lipid region is not significantly different. However, within the 50 to $500 \mathrm{~m} / \mathrm{z}$ range (typically fatty acids and lower molecular weight metabolites) there is a significant increase for all storage conditions at 72 hours, with the largest increase seen at room temperature. This increase is in line with previous work on the metabolic profiling, albeit using $1 \mathrm{H}$ nuclear magnetic resonance spectroscopy, of human faecal samples which found that storage at room temperature substantially affected fatty acids and low 
molecular weight metabolites, but that storage at $4^{\circ} \mathrm{C}$ or below reduced this effect ${ }^{28}$ - likely as a result of reduced microbial metabolism of complex lipids.

\section{Effect of Freeze/Thaw Cycles on REIMS Mass Spectra}

In addition to storage condition and length, the effect of consecutive freeze/thaw cycles on the REIMS mass spectra was assessed. For this assessment, one portion of participants' faecal samples was frozen at $-80^{\circ} \mathrm{C}$ for two hours, thawed at room temperature for one hour and analysed using the highthroughput, monopolar REIMS platform as previously described. For each sample, a total of six freeze/thaw cycles, with REIMS analysis after each, was completed. Unsupervised PCA modelling shows a clear trend towards separation of samples, from all participants, according to freeze/thaw cycle, Figure 3c. Univariate, non-parametric statistical analysis using Kruskal-Wallis one-way analysis of variance identified all significant, FDR-corrected $P$ value below 0.001 , mass bins, between 50 to $2500 \mathrm{~m} / \mathrm{z}$, different between freeze/thaw cycles, Supplementary Figure $\mathrm{s} 7$. The majority of significantly different $\mathrm{m} / \mathrm{z}$ bins are below $300 \mathrm{~m} / \mathrm{z}$ or above $1100 \mathrm{~m} / \mathrm{z}$ suggesting that molecular species such as fatty acids, and dimers of diglyceride and glycerophospholipid which are of high intensity within these respective ranges are more susceptible to freeze/thaw-induced breakdown. The stability of the 600 to $1000 \mathrm{~m} / \mathrm{z}$ range was confirmed through unsupervised PCA modelling, Figure 3d, which shows no significant separation of samples based on freeze/thaw cycles. Additionally, intensity analysis shows significant increases in both noise $(50$ to $51 \mathrm{~m} / \mathrm{z}$ ) and the 50 to $500 \mathrm{~m} / \mathrm{z}$ range, particularly after three of more freeze/thaw cycles. Conversely, the complex lipid region of 600 to $1000 \mathrm{~m} / \mathrm{z}$ range shows no significant differences in signal intensity of any freeze/thaw cycle compared to fresh faecal samples. Unsupervised PCA modelling using this restricted mass range, Figure 4a, shows that clear and significant separation is evident between participants within this mass range, using mass spectra from all six freeze/thaw cycles; with reduced separation evident between participants using the 50 to $500 \mathrm{~m} / \mathrm{z}$ range evident. This difference in separation may be indicative of two factors. Firstly, 
information from the complex lipid region (600 to $1000 \mathrm{~m} / \mathrm{z}$ ) rather than the fatty acid and lower molecular weight metabolite region (50 to $500 \mathrm{~m} / \mathrm{z}$ ). Arguably, the complex lipid region holds more scope for biochemical differences to emerge as a result of the wide range of detectable combinations of head groups and acyl chains. Secondly, it may support the conclusions drawn from Figure 3 that the complex lipid region is more stable through successive freeze/thaw cycles than the lower mass range and is therefore, better able to preserve individual differences that are detectable through REIMS. This would further support the benefit of using REIMS for direct-from-sample metabolomics of faeces over traditional mass spectrometry approaches as it does not require the storage and bulk processing of samples that is common in techniques such as LC-MS and GC-MS. 
decisions. In addition to bacterial-derived metabolites of bile acids, we have also identified a number of bacteria-associated metabolites in faecal samples including cresol, butanal, mandelic acid, and urocanic acid.

In addition to bile acids, phospholipids, which constituted a large proportion of the REIMS mass spectra generated, were identified directly from faecal samples using tandem mass spectrometry, Table 1. The dominant phospholipid class identified using this method, phosphatidylglycerol (PG), is the main lipid component, alongside phosphatidylethanolamine (PE), of the bacterial inner cell membrane. Using REIMS, we have previously shown that these lipid classes hold substantial specieslevel discriminatory power between clinically important microorganisms ${ }^{21,22}$ and could thus serve as biomarkers for constituents of the faecal microbiome. Supporting this, three of the four identified PG lipids contained odd-chain, 15:0, acyl chains, which are indicative of bacterial lipids ${ }^{31}$, although, it should be noted that odd-chain acyl chains and free fatty acids are also associated with ruminant metabolism ${ }^{32}$, and thus may be indicative of dietary intake. Nonetheless, REIMS could serve as a tool for the rapid identification of pathobiont species directly from sample. In addition, REIMS may also allow for a rapid and non-invasive method for the screening of faecal samples to monitor shifts in metabolic content which may be diagnostic of disease status and/or onset. In addition to those features identified using tandem mass spectrometry, a total of 169 features were tentatively identified, Supplementary Data Matrix 1, across a wide range of classes, using accurate mass measurements. This data shows the wide range of compounds which can be detected using REIMS, including amino acids and peptides, in human faeces.

\section{Application of Optimised REIMS Parameters to Clinical Faecal Samples}

Bariatric surgery is performed on patients with severe obesity to bring about profound weight loss. and can lead to improvements in comorbidities such as type two diabetes mellitus, hypertension, and obstructive sleep apnoea ${ }^{33}$. Although the marked effect of bariatric surgery on the human gut 
microbiome has been extensively studied ${ }^{34}, 35$, there is limited understanding of how the faecal metabolome is altered. NMR-based spectroscopy of serum shows that the wider human metabolome is affected by bariatric surgery ${ }^{36}$, suggesting that concomitant changes to the faecal metabolome are likely and these should be detectable using REIMS.

To show that REIMS is capable of detecting biological differences using the optimised parameters identified herein, a cohort of 50 faecal samples from patients pre-, two to six months post-, and one to two years post-bariatric surgery were analysed. REIMS analysis was carried out on these samples, and following data pre-processing as previously described, the 50 to $1200 \mathrm{~m} / \mathrm{z}$ range at $0.1 \mathrm{Da}$ bins was used for statistical analysis. From both unsupervised and supervised multivariate modelling, Figure 6, a clear separation is evident between the pre- and one to two years post-surgery faecal samples, with those collected one to six months post-surgery showing an intermediary distribution between the two time points. Analysis of the features contributing towards this observed separation, Supplementary Figure S7, show that the primary drivers are present within the 600 to $1200 \mathrm{~m} / \mathrm{z}$ range, with a high number of important features between 1000 and $1200 \mathrm{~m} / \mathrm{z}$. Furthermore, univariate analysis of variance tests also shows that the most significantly different features between the three time points are above $600 \mathrm{~m} / \mathrm{z}$, albeit with a substantial number below this cut-off. Supplementary Table S4 shows tentative identifications of significant features driving the observed separation in Figure 6. Based on accurate mass determination, these tentative identifications support the observation that there are significant changes in faecal lipid composition, namely phospholipids including $\mathrm{PC}(44: 3), \mathrm{PE}(46: 0), \mathrm{PC}(42: 0), \mathrm{PE}(\mathrm{P}-38: 0)$, and $\mathrm{PE}(46: 1)$ and triglycerides $\mathrm{TG}(54: 3), \mathrm{TG}(58: 0)$, and $\mathrm{TG}(52: 4)$ as a result of bariatric surgery. Unsurprisingly, alterations in lipid concentrations following bariatric surgery, as a result of reduced dietary intake, are common ${ }^{37,}{ }^{38}$. Indeed, dyslipidaemia, a condition linked to cardiovascular disease and stroke, resulting in abnormal composition of lipids within the body, is often resolved following bariatric surgery ${ }^{39}$. Thus, REIMS is 

capable of detecting important biological changes following a clinical intervention - proving its utility as a rapid, direct-from-sample metabolic profiling platform for human faeces. 
As an alternative to traditional metabolic profiling approaches which have linked mass spectrometry with a pre-analysis chromatographic separation, REIMS offers a platform for the high-throughput, automated, and low-cost analysis of biological material. As well as the benefit of faster analysis, resulting from the removal of the requirement for sample preparation, REIMS allows the analysis of a sample, such as faeces, in its natural state. These properties present a number of novel application areas in which this proof-of-concept work could be extended. For example, the biochemical analysis of faeces, including metabolite profiling, has been suggested as a potential approach for either whole or targeted population disease screening programmes, such as for colorectal cancer. Due to its highthroughput, automated, and low-cost nature, REIMS may provide a practical alternative to existing methods such as Cologuard ${ }^{40}$, which can be time-consuming and costly. This ability would allow for the screening of a large number of patient samples, and would not be limited to one, or a low number of target compounds. Although this work utilised the REIMS analysis of human faeces, a wide-range of biofluids could also be analysed using the technology, such as sputum or urine, allowing a single platform to be used for the screening of a diverse range of diseases e.g. cystic fibrosis and urinary tract infections. One of the main constituents of human faeces is microbial, and although the gut microbiome is an essential component of human physiology, pathogenic microorganisms, such as Clostridioides difficile, are capable of causing disease. Current techniques require culturing of a sample to identify the causative organism of an infection, which is labour intensive and time-consuming. As we have suggested, REIMS may be capable of detecting bacterial phospholipids, which might provide a novel diagnostic method that allows direct-from-sample detection of pathogens. This would however, require additional work to determine the level of taxonomic specificity which could be achieved. 
Detailed information regarding bacterial and fungal species analysed in this study, and on additional data analyses completed as referenced within this text is given within the supporting information accompanying this manuscript. This material is available free of charge via the Internet at http://pubs.acs.org.

\section{Author Contributions}

Study was planned by SJSC, JLA, JVL, and ZT. Experiments were conducted by SJSC, JA, HA, and JVL.

Data was analysed and interpreted by SJSC, JLA, and ZT. Technical assistance was provided by FB, AB, JT, JRM, and JK. The manuscript was written by SJSC, JLA, and ZT with input from all authors. All authors have given approval to the final version of the manuscript.

\section{Conflict of Interest Statement}

This work was funded and technically supported by the Waters Corporation and funded by the Biotechnology and Biological Sciences Research Council under grant BB/L020858/1 and European Research Council under contract number 617896. Z.T. provides remunerated consultancy to the Waters Corporation. The work detailed in this manuscript does not promote any available commercial product from Waters Corporation.

\section{Acknowledgments}

413 The authors would like to thank Imperial College Healthcare Trust for access to samples and to the 414 participants/patients who agreed to donate samples which were utilised in this work. We also 415 acknowledge support from Imperial College NIHR Biomedical Research Centre and Bowel and Cancer 416 Research. This work was funded and technically supported by the Waters Corporation and funded by the Biotechnology and Biological Sciences Research Council under grant BB/L020858/1 and European 
419 NIHR BRC, and the views expressed in this publication are those of the authors and not necessarily 420 those of the NHS, NIHR, or the Department of Health. 
422 1. Dettmer, K.; Aronov, P. A.; Hammock, B. D., Mass Spectrometry-Based Metabolomics. Mass Spectrometry Reviews 2007, 26 (1), 51-78.

2. Nicholson, J. K.; Holmes, E.; Kinross, J.; Burcelin, R.; Gibson, G.; Jia, W.; Pettersson, S., Hostgut microbiota metabolic interactions. Science 2012, 336 (6086), 1262-1267.

3. Moloney, R. D.; Desbonnet, L.; Clarke, G.; Dinan, T. G.; Cryan, J. F., The microbiome: stress, health and disease. Mammalian genome 2014, 25 (1-2), 49-74.

4. Shreiner, A. B.; Kao, J. Y.; Young, V. B., The gut microbiome in health and in disease. Current opinion in gastroenterology 2015, 31 (1), 69.

5. Zhang, T.; Watson, D. G., A short review of applications of liquid chromatography mass spectrometry based metabolomics techniques to the analysis of human urine. Analyst 2015,

6. Zhang, A.; Sun, H.; Yan, G.; Wang, P.; Wang, X., Mass spectrometry-based metabolomics: applications to biomarker and metabolic pathway research. Biomedical Chromatography 2016,

7. Armitage, E. G.; Barbas, C., Metabolomics in cancer biomarker discovery: current trends and future perspectives. Journal of pharmaceutical and biomedical analysis 2014, 87, 1-11.

8. Cameron, S. J.; Lewis, K. E.; Beckmann, M.; Allison, G. G.; Ghosal, R.; Lewis, P. D.; Mur, L. A., The Metabolomic Detection of Lung Cancer Biomarkers in Sputum. Lung Cancer 2016, 94, 8895.

9. Cheng, Y.-S. L.; Rees, T.; Wright, J., A review of research on salivary biomarkers for oral cancer detection. Clinical and translational medicine 2014, 3 (1), 3.

10. Weir, T. L.; Manter, D. K.; Sheflin, A. M.; Barnett, B. A.; Heuberger, A. L.; Ryan, E. P., Stool microbiome and metabolome differences between colorectal cancer patients and healthy adults. PloS one 2013, 8 (8), e70803. 
11. Morgan, X. C.; Tickle, T. L.; Sokol, H.; Gevers, D.; Devaney, K. L.; Ward, D. V.; Reyes, J. A.; Shah, S. A.; LeLeiko, N.; Snapper, S. B., Dysfunction of the intestinal microbiome in inflammatory bowel disease and treatment. Genome biology 2012, 13 (9), R79.

12. Gika, H. G.; Theodoridis, G. A.; Plumb, R. S.; Wilson, I. D., Current practice of liquid chromatography-mass spectrometry in metabolomics and metabonomics. Journal of pharmaceutical and biomedical analysis 2014, 87, 12-25.

13. Pasikanti, K. K.; Ho, P.; Chan, E., Gas chromatography/mass spectrometry in metabolic profiling of biological fluids. Journal of Chromatography B 2008, 871 (2), 202-211.

14. Maurer, H. H., Hyphenated mass spectrometric techniques-indispensable tools in clinical and forensic toxicology and in doping control. Journal of Mass Spectrometry 2006, 41 (11), 13991413.

15. Maharjan, R. P.; Ferenci, T., Global metabolite analysis: the influence of extraction methodology on metabolome profiles of Escherichia coli. Analytical biochemistry 2003, 313 (1), 145-154.

16. Monge, M. E.; Harris, G. A.; Dwivedi, P.; Fernández, F. M., Mass spectrometry: recent advances in direct open air surface sampling/ionization. Chemical reviews 2013, 113 (4), 2269-2308.

17. Balog, J.; Sasi-Szabó, L.; Kinross, J.; Lewis, M. R.; Muirhead, L. J.; Veselkov, K.; Mirnezami, R.; Dezső, B.; Damjanovich, L.; Darzi, A.; Nicholson, J. K.; Takats, Z., Intraoperative tissue identification using rapid evaporative ionization mass spectrometry. Sci. Transl. Med. 2013, 5, 194ra93-194ra93.

18. Strittmatter, N.; Jones, E. A.; Veselkov, K. A.; Rebec, M.; Bundy, J. G.; Takats, Z., Analysis of Intact Bacteria using Rapid Evaporative Ionisation Mass Spectrometry. Chem. Commun. 2013, $49,6188-6190$.

19. Strittmatter, N.; Rebec, M.; Jones, E. A.; Golf, O.; Abdolrasouli, A.; Balog, J.; Behrends, V.; Veselkov, K. A.; Takats, Z., Characterization and identification of clinically relevant microorganisms using rapid evaporative ionization mass spectrometry. Anal. Chem. 2014, 86, 6555-6562. 
20. Schäfer, K. C.; Dénes, J.; Albrecht, K.; Szaniszló, T.; Balog, J.; Skoumal, R.; Katona, M.; Tóth, M.; Balogh, L.; Takáts, Z., In vivo, in situ tissue analysis using rapid evaporative ionization mass spectrometry. Angewandte Chemie International Edition 2009, 48 (44), 8240-8242.

21. Bolt, F.; Cameron, S. J.; Karancsi, T.; Simon, D.; Schaffer, R.; Rickards, T.; Hardiman, K.; Burke, A.; Bodai, Z.; Perdones-Montero, A.; Rebec, M.; Balog, J.; Takats, Z., Automated HighThroughput Identification and Characterization of Clinically Important Bacteria and Fungi using Rapid Evaporative Ionization Mass Spectrometry. Analytical Chemistry 2016, 88 (19), 94199426.

22. Cameron, S. J.; Bolt, F.; Perdones-Montero, A.; Rickards, T.; Hardiman, K.; Abdolrasouli, A.; Burke, A.; Bodai, Z.; Karancsi, T.; Simon, D.; Schaffer, R.; Rebec, M.; Balog, J.; Takats, Z., Rapid Evaporative Ionisation Mass Spectrometry (REIMS) Provides Accurate Direct from Culture Species Identification within the Genus Candida. Scientific Reports 2016, 6, 36788.

23. Lewis, S.; Heaton, K., Stool form scale as a useful guide to intestinal transit time. Scandinavian journal of gastroenterology 1997, 32 (9), 920-924.

24. Xia, J.; Sinelnikov, I. V.; Han, B.; Wishart, D. S., MetaboAnalyst 3.0 - Making Metabolomics More Meaningful. Nucleic Acids Research 2015, 43 (W1), W251-257.

25. Wishart, D. S.; Jewison, T.; Guo, A. C.; Wilson, M.; Knox, C.; Liu, Y.; Djoumbou, Y.; Mandal, R.; Aziat, F.; Dong, E.; Bouatra, S.; Sinelnikov, I.; Arndt, D.; Xia, J.; Liu, P.; Yallou, F.; Bjorndahl, T.; Perez-Pineiro, R.; Eisner, R.; Allen, F.; Neveu, V.; Greiner, R.; Scalbert, A., HMDB 3.0: The Human Metabolome Database in 2013. Nucleic Acids Research 2013, 41 (Database issue), D8017.

26. Fahy, E.; Subramaniam, S.; Murphy, R. C.; Nishijima, M.; Raetz, C. R. H.; Shimizu, T.; Spener, F.; van Meer, G.; Wakelam, M. J. O.; Dennis, E. A., Update of the LIPID MAPS comprehensive classification system for lipids. Journal of Lipid Research 2009, 50, S9-S14.

27. Bodai, Z.; Cameron, S.; Bolt, F.; Simon, D.; Schaffer, R.; Karancsi, T.; Balog, J.; Rickards, T.; Burke, A.; Hardiman, K., Effect of Electrode Geometry on the Classification Performance of 
Rapid Evaporative Ionization Mass Spectrometric (REIMS) Bacterial Identification. Journal of The American Society for Mass Spectrometry 2017, 1-8.

28. Gratton, J.; Phetcharaburanin, J.; Mullish, B. H.; Williams, H. R.; Thursz, M.; Nicholson, J. K.; Holmes, E.; Marchesi, J. R.; Li, J. V., Optimized Sample Handling Strategy for Metabolic Profiling of Human Feces. Analytical chemistry 2016, 88 (9), 4661-4668.

29. Duboc, H.; Rajca, S.; Rainteau, D.; Benarous, D.; Maubert, M.-A.; Quervain, E.; Thomas, G.; Barbu, V.; Humbert, L.; Despras, G., Connecting dysbiosis, bile-acid dysmetabolism and gut inflammation in inflammatory bowel diseases. Gut 2013, 62 (4), 531-539.

30. Kakiyama, G.; Pandak, W. M.; Gillevet, P. M.; Hylemon, P. B.; Heuman, D. M.; Daita, K.; Takei, H.; Muto, A.; Nittono, H.; Ridlon, J. M., Modulation of the fecal bile acid profile by gut microbiota in cirrhosis. Journal of hepatology 2013, 58 (5), 949-955.

31. Hopkins, M.; Sharp, R.; Macfarlane, G., Age and disease related changes in intestinal bacterial populations assessed by cell culture, $16 \mathrm{~S}$ rRNA abundance, and community cellular fatty acid profiles. Gut 2001, 48 (2), 198-205.

32. Jenkins, B.; West, J.; Koulman, A., A review of odd-chain fatty acid metabolism and the role of pentadecanoic acid (C15: 0) and heptadecanoic acid (C17: 0) in health and disease. Molecules 2015, 20 (2), 2425-2444.

33. Buchwald, H.; Avidor, Y.; Braunwald, E.; Jensen, M. D.; Pories, W.; Fahrbach, K.; Schoelles, K., Bariatric surgery: a systematic review and meta-analysis. Jama 2004, 292 (14), 1724-1737.

34. Graessler, J.; Qin, Y.; Zhong, H.; Zhang, J.; Licinio, J.; Wong, M.-L.; Xu, A.; Chavakis, T.; Bornstein, A.; Ehrhart-Bornstein, M., Metagenomic sequencing of the human gut microbiome before and after bariatric surgery in obese patients with type 2 diabetes: correlation with inflammatory and metabolic parameters. The pharmacogenomics journal 2013, 13 (6), 514-522.

35. Furet, J.-P.; Kong, L.-C.; Tap, J.; Poitou, C.; Basdevant, A.; Bouillot, J.-L.; Mariat, D.; Corthier, G.; Doré, J.; Henegar, C., Differential adaptation of human gut microbiota to bariatric surgeryinduced weight loss. Diabetes 2010, 59 (12), 3049-3057. 
36. Gralka, E.; Luchinat, C.; Tenori, L.; Ernst, B.; Thurnheer, M.; Schultes, B., Metabolomic fingerprint of severe obesity is dynamically affected by bariatric surgery in a proceduredependent manner. The American journal of clinical nutrition 2015, 102 (6), 1313-1322.

37. Brolin, R. E.; Bradley, L. J.; Wilson, A. C.; Cody, R. P., Lipid risk profile and weight stability after gastric restrictive operations for morbid obesity. Journal of Gastrointestinal Surgery 2000, 4 (5), 464-469.

38. Strain, G. W.; Saif, T.; Ebel, F.; Dakin, G. F.; Gagner, M.; Costa, R.; Chiu, Y.-L.; Pomp, A., Lipid profile changes in the severely obese after laparoscopic sleeve gastrectomy (LSG), 1, 3, and 5 years after surgery. Obesity surgery 2015, 25 (2), 285-289.

39. Courcoulas, A. P.; Christian, N. J.; Belle, S. H.; Berk, P. D.; Flum, D. R.; Garcia, L.; Horlick, M.; Kalarchian, M. A.; King, W. C.; Mitchell, J. E., Weight change and health outcomes at 3 years after bariatric surgery among individuals with severe obesity. Jama 2013, 310 (22), 2416-2425.

40. Imperiale, T. F.; Ransohoff, D. F.; Itzkowitz, S. H.; Levin, T. R.; Lavin, P.; Lidgard, G. P.; Ahlquist, D. A.; Berger, B. M., Multitarget stool DNA testing for colorectal-cancer screening. N Engl J Med 2014, 2014 (370), 1287-1297. 
541 Phospholipids identified through interrogation of LIPID MAPS database for possible species for highest

542 intensity $\mathrm{m} / \mathrm{z}$ bins, followed by tandem mass spectrometry fragmentation with accurate mass

543 identification of potential acyl chains.

544

\begin{tabular}{|c|c|c|c|c|c|c|c|}
\hline \multicolumn{5}{|c|}{ Lipid Candidate } & \multicolumn{3}{|c|}{ MS/MS Fatty Acid } \\
\hline$m / z$ Bin & ID & $\begin{array}{l}\text { Theoretical } \\
\text { Mass }\end{array}$ & Delta Value & Ion & ID & $m / z$ Fragment & Delta Value \\
\hline \multirow{2}{*}{653.51} & \multirow{2}{*}{ DG(39:6) } & \multirow{2}{*}{653.5150} & \multirow{2}{*}{0.0050} & \multirow{2}{*}[\mathrm{M}-\mathrm{H}]{-} & $15: 0$ & 241.23 & 0.0127 \\
\hline & & & & & $24: 6$ & 397.12 & 0.0820 \\
\hline \multirow{2}{*}{691.49} & \multirow{2}{*}{$P G(P-31: 0)$} & \multirow{2}{*}{691.4919} & \multirow{2}{*}{0.0019} & \multirow{2}{*}[\mathrm{M}-\mathrm{H}]{-} & $16: 0$ & 255.25 & 0.0171 \\
\hline & & & & & $15: 0$ & 241.23 & 0.0127 \\
\hline \multirow{2}{*}{717.51} & \multirow{2}{*}{ PG(P-33:1) } & \multirow{2}{*}{717.5076} & \multirow{2}{*}{0.0024} & \multirow{2}{*}[\mathrm{M}-\mathrm{H}]{-} & $18: 1$ & 281.27 & 0.0214 \\
\hline & & & & & $15: 0$ & 241.24 & 0.0227 \\
\hline \multirow{2}{*}{731.52} & \multirow{2}{*}{ PG(0-34:1) } & \multirow{2}{*}{731.5232} & \multirow{2}{*}{0.0032} & \multirow{2}{*}[\mathrm{M}-\mathrm{H}]{-} & $18: 1$ & 281.27 & 0.0214 \\
\hline & & & & & $16: 0$ & 255.25 & 0.0171 \\
\hline
\end{tabular}


547 A modified TECAN Freedom Evoware 75 robotic platform was used for automated REIMS analysis of

548 faecal samples. For analysis (a) a modified robotic tip with a sharp monopolar electrode makes contact

549 with the faecal sample and generates a radiofrequency electrical current. Due to the non-zero

550 impedance of the sample, this results in rapid heating of the sample and the generation of an aerosol

551 containing gas phase ions. This aerosol is transferred directly to a mass spectrometer via an

552 atmospheric interface where it combines with a solvent matrix and collides with a heated collision

553 surface, resulting in the formation of individual ion species which enter the ion guide of the

554 instrument. REIMS analysis allows for the detection of both (b) negative ion and (c) positive ion

555 molecular species across the mass range of 50 to $2500 \mathrm{~m} / \mathrm{z}$. Mass spectra shown after background

556 subtraction and lock mass correction using MassLynx software.

557

(a) Analytical Set-Up

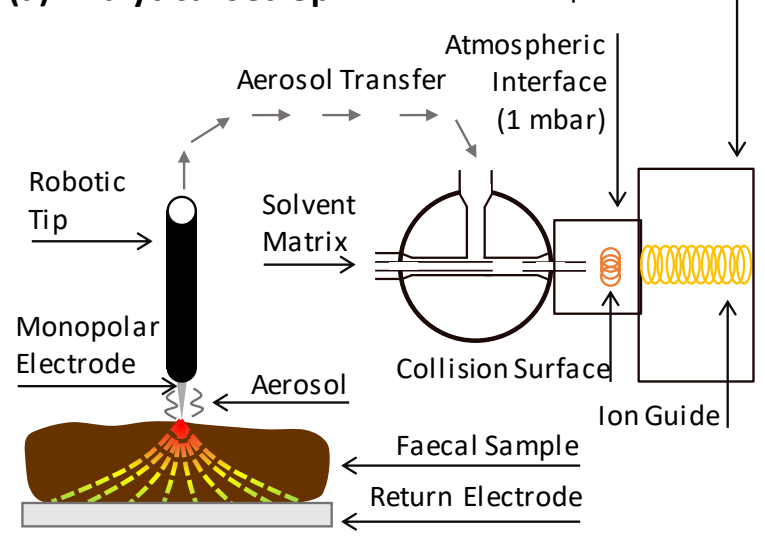

(b) Negative Ion Detection

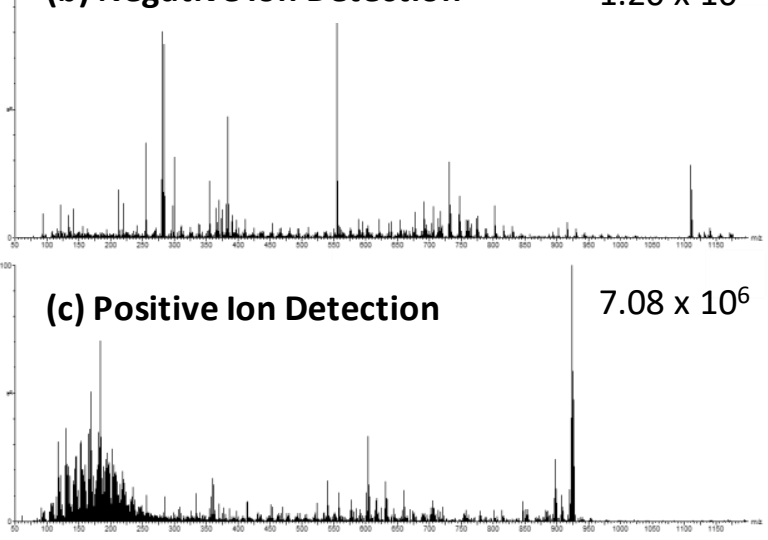


Heating power optimisation was completed using power levels between $10 \mathrm{~W}$ and $50 \mathrm{~W}$, increasing in

(a) Region of Interest Signal Intensities

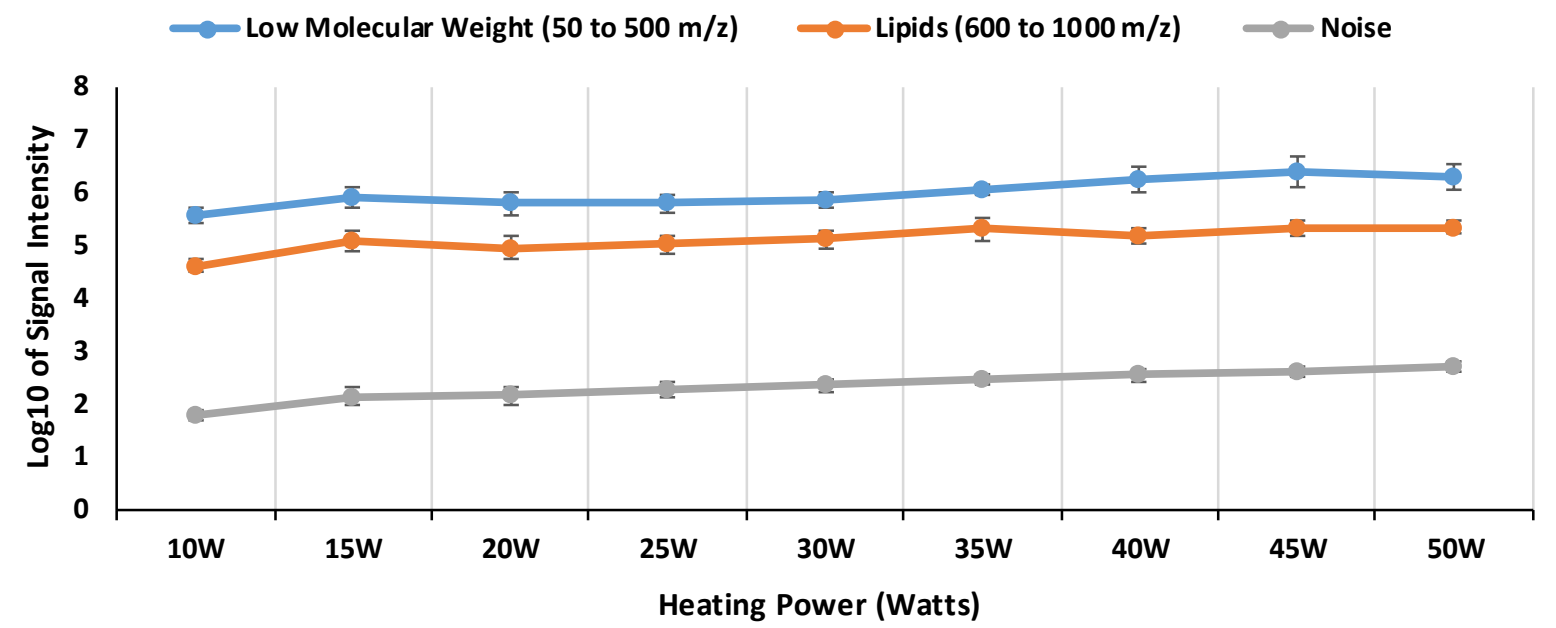

(b) Heating Power

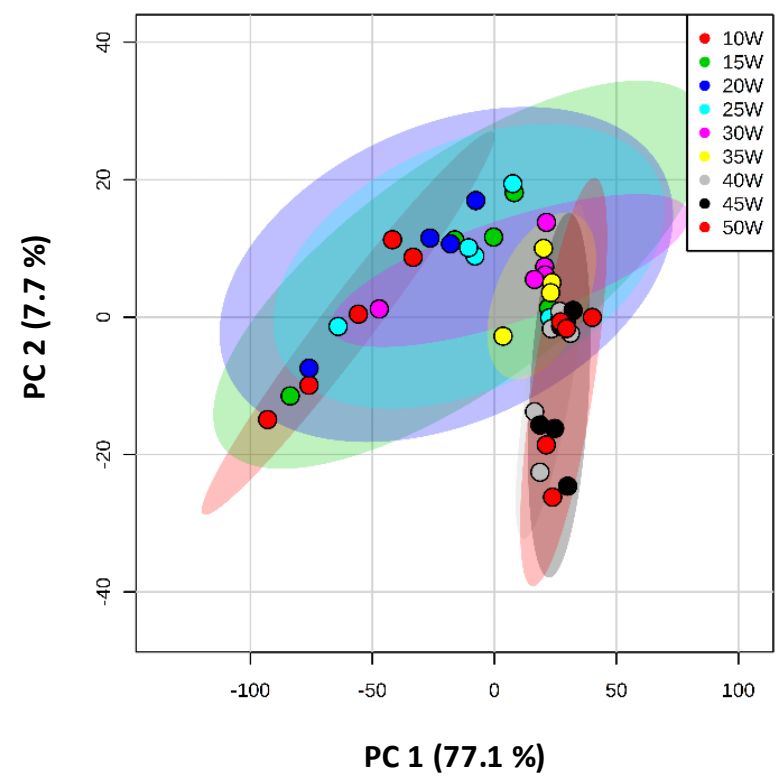

(c) Participant

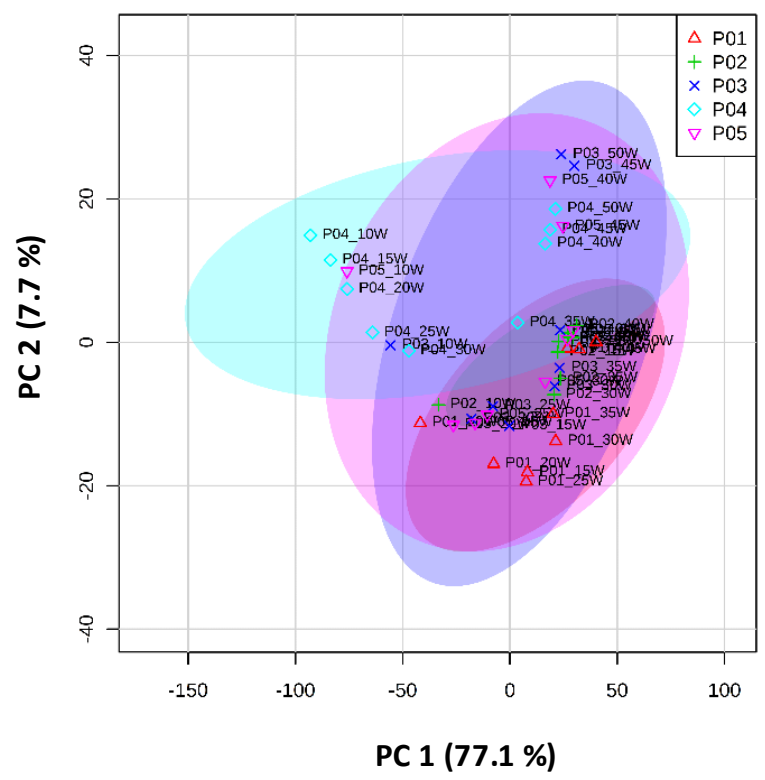


567 (a) Principal component analysis over the 50 to $2500 \mathrm{~m} / \mathrm{z}$ range shows no significant separation of storage conditions, with (b) hierarchical cluster analysis shows participant differences using the 25 most significant mass bins regardless of storage conditions. PCA plots within the (c) 50 to $500 \mathrm{~m} / \mathrm{z}$ range and (d) 600 to $1000 \mathrm{~m} / \mathrm{z}$ range show significant effects of successive freeze/thaw cycles within

571 the lower molecular weight region, but not within the complex lipid region. Shaded colour areas

572 represent $95 \%$ confidence intervals of significant separation. Intensity analysis and univariate analysis

573 is given in Supplementary Figures S6 and S7.

(a) Storage Condition

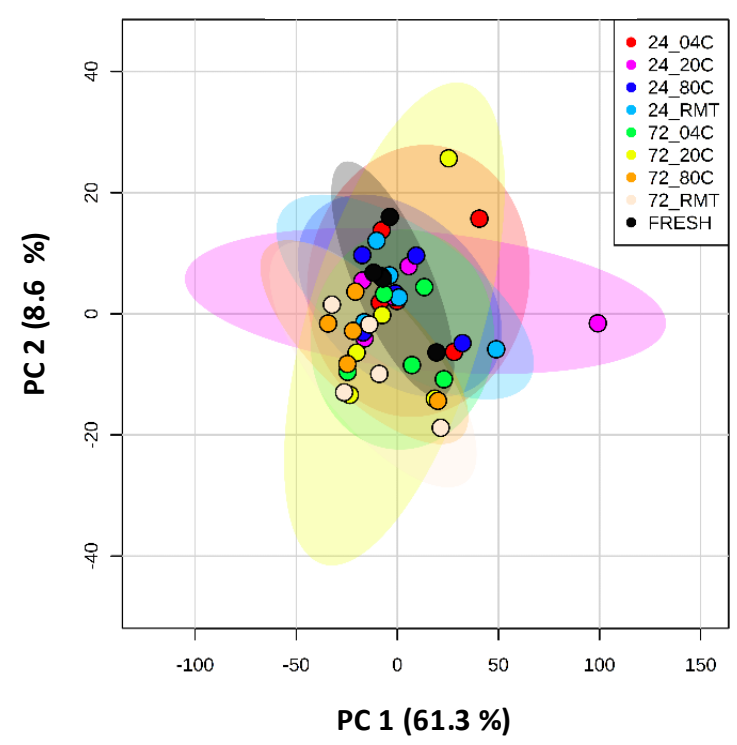

(c) 50 to $500 \mathrm{~m} / \mathrm{z}$ Range

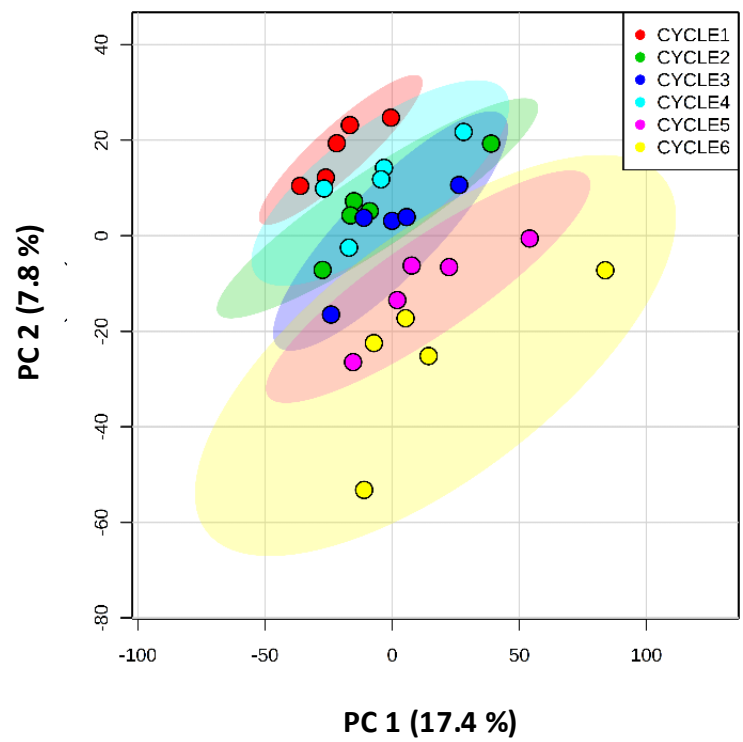

(b) Participant

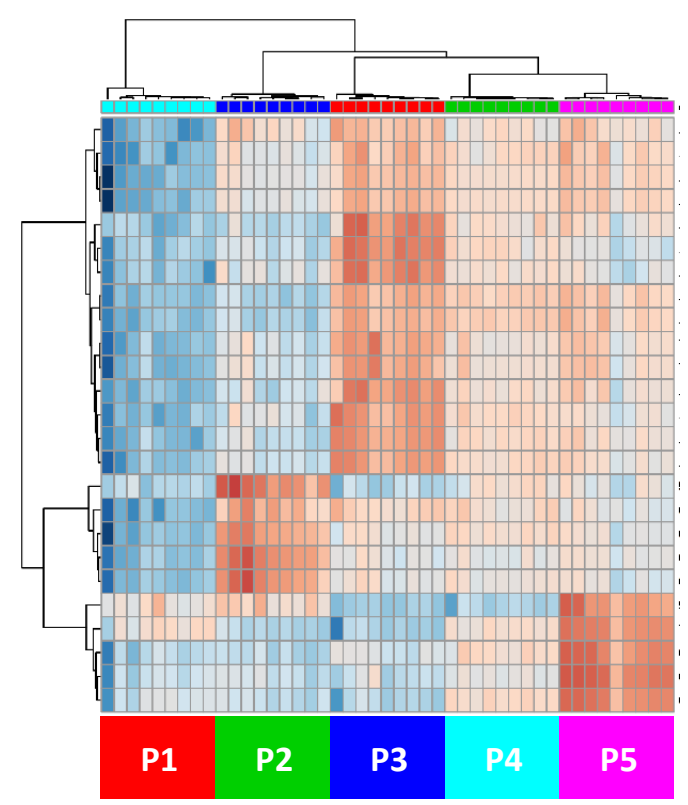

(d) 600 to $1000 \mathrm{~m} / \mathrm{z}$ Range

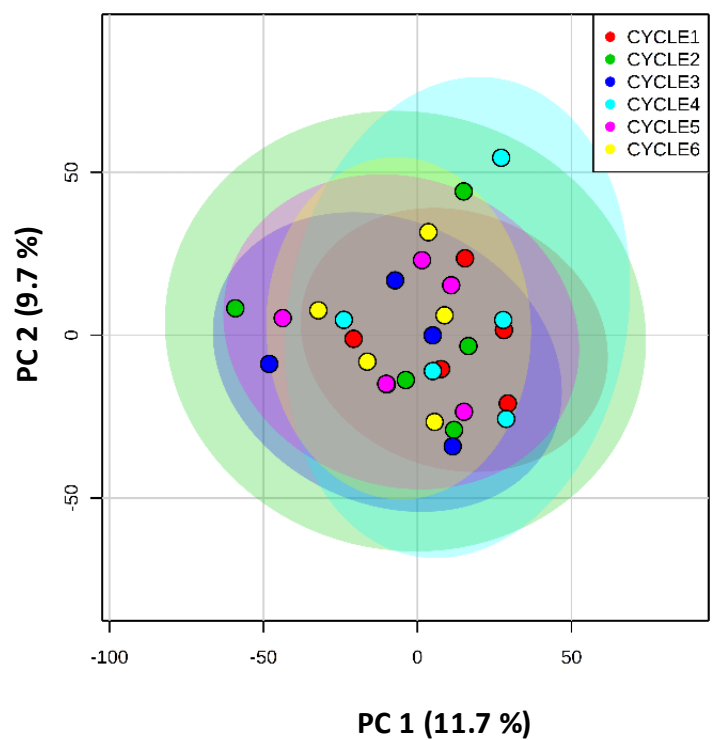



groupings. Cycle Key: $\boldsymbol{\Delta}=1 ; \boldsymbol{\square}=2 ; \bullet=3 ; \bullet=4 ; \bullet \bullet=5 ; \boldsymbol{\nabla}=6$;

579

(a) 600 to $1000 \mathrm{~m} / \mathrm{z}$ Range

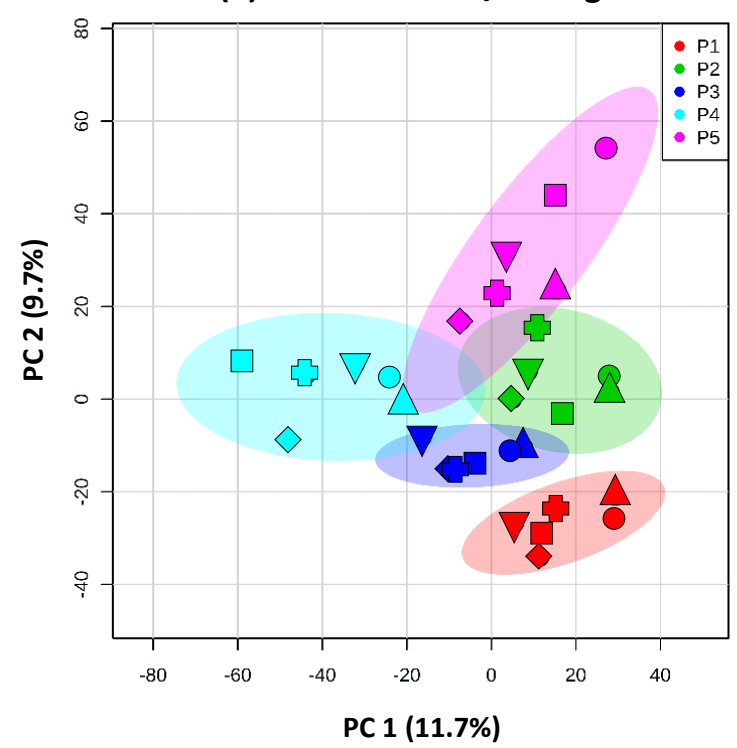

(b) 50 to $500 \mathrm{~m} / \mathrm{z}$ Range

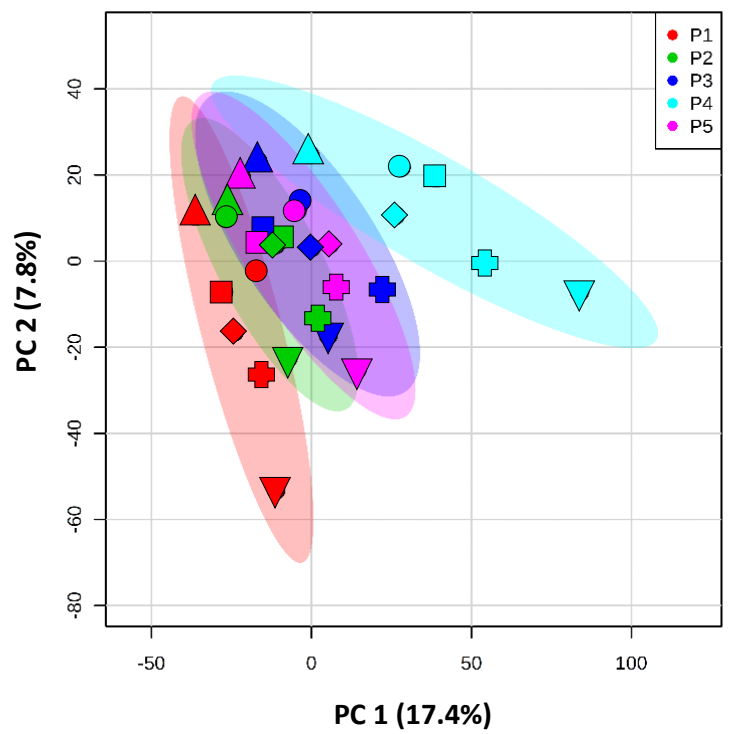


Annotated mass spectra for one participant of (a) bile acids tentatively identified through accurate
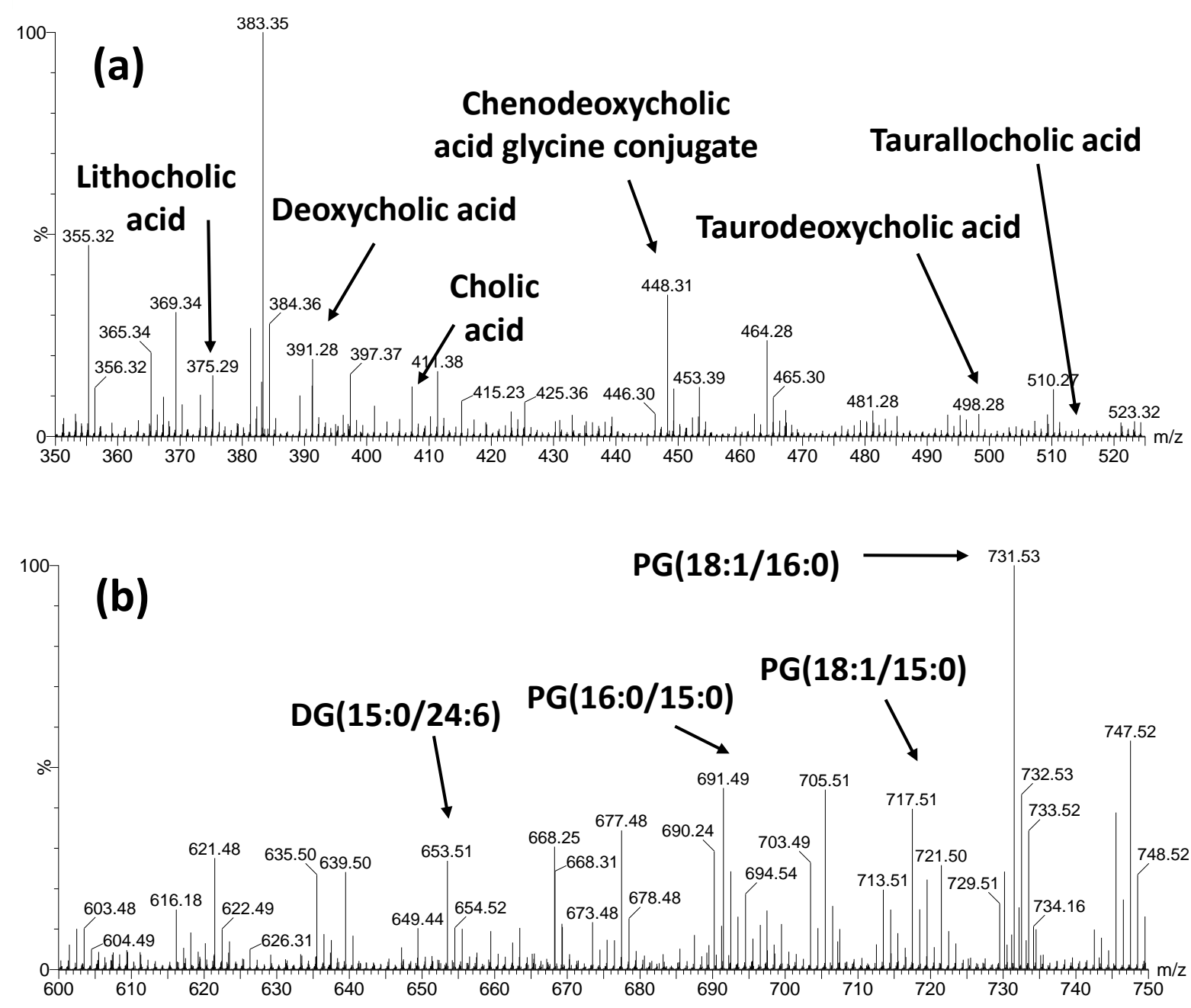
Modelling of the 50 to $1200 \mathrm{~m} / \mathrm{z}$ range using (a) principal component modelling and (b) partial least square discriminant analysis shows that there is a significant separation between time points. Shaded areas show $95 \%$ confidence intervals of each grouping. Performance scores for PLS-DA model in (b) using ten-fold cross-validation, are Accuracy $=0.70 ; R 2=0.80 ; \mathrm{Q} 2=0.55$. PCA loading and importance feature plots are show in Supplementary Figure S8. Univariate analysis using (c) one-way Kruskalin Supplementary Table S4.

(a) PCA Plot

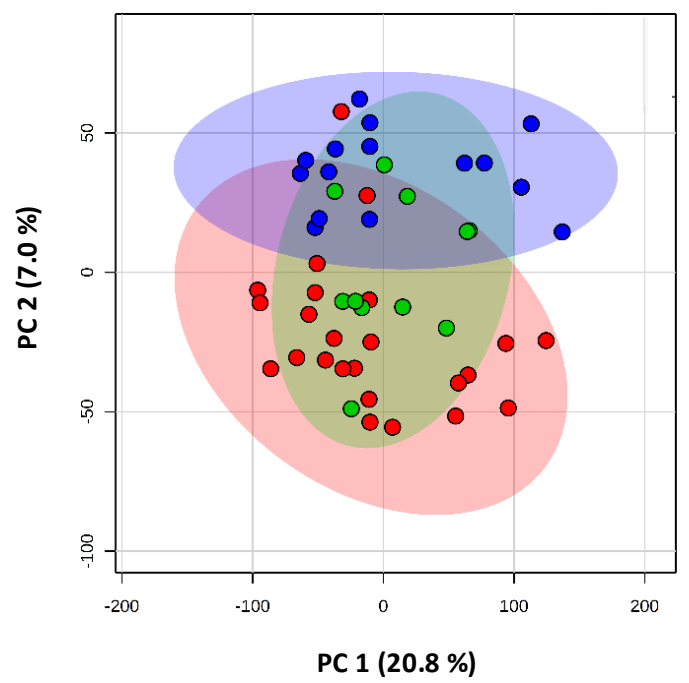

(b) PLS-DA Plot

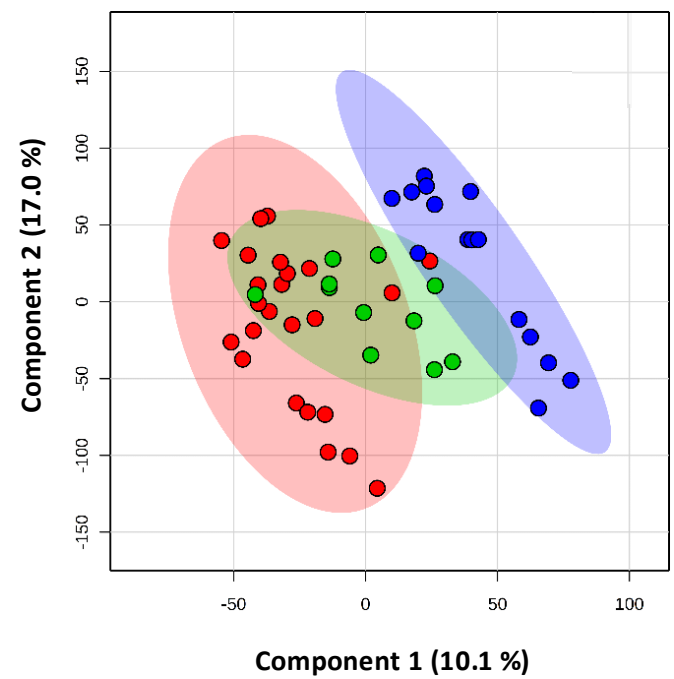

Before Surgery

(c) Significant Features between Timepoints through Kruskal-Wallis Analysis

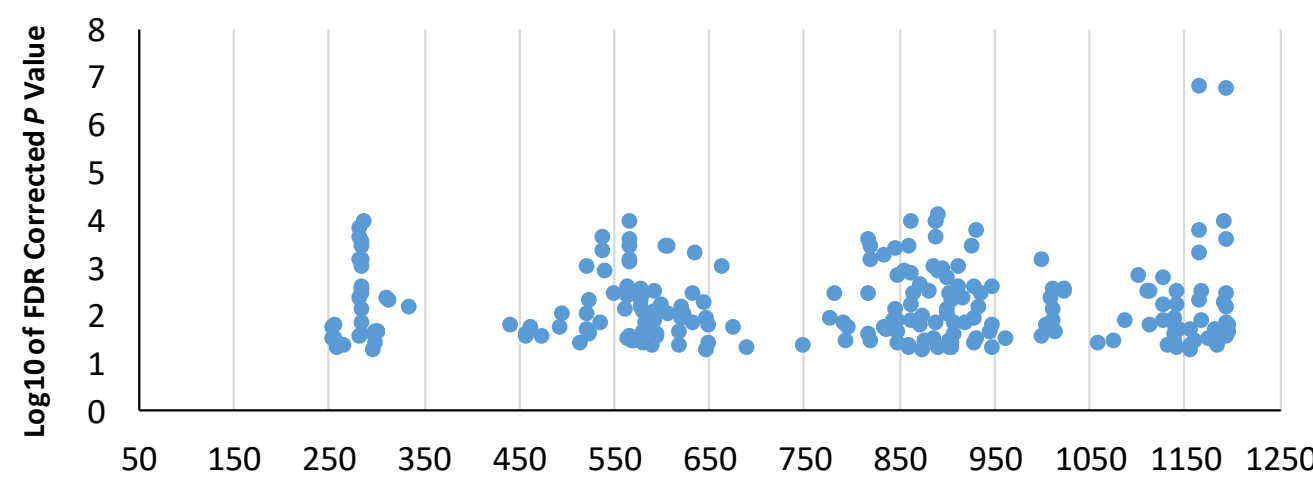

$m / z$ Value of Significant Feature 
597

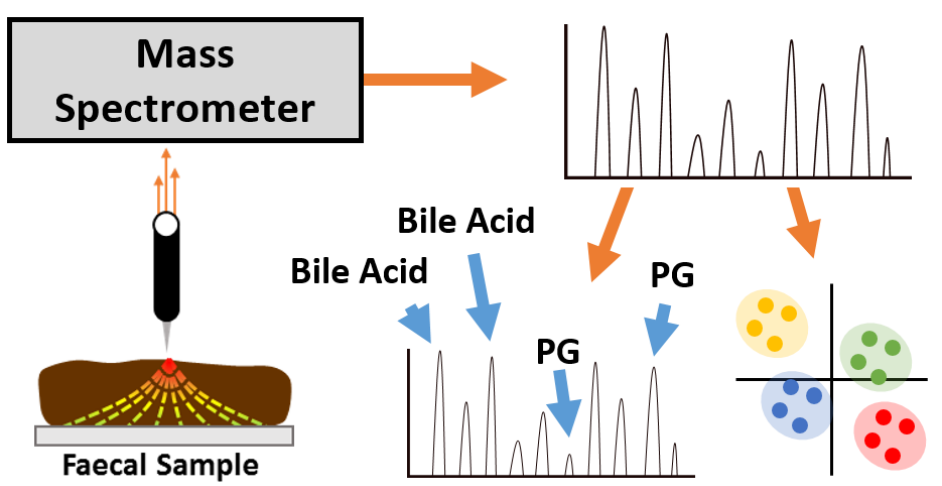

598

599 The first application of rapid evaporative ionisation mass spectrometry, requiring no sample 600 preparation, to the analysis of the human faecal metabolome. 